\title{
Mechanisms of nanosecond pulsed electric field (nspef)-induced cell death in cells and tumors
}

\begin{abstract}
The evolution of pulse power technology from high power physics to biology and medicine places nanosecond pulsed electric fields (nsPEFs) in positions for in vitro and in vivo applications as non-ligand agonists that not only bypass plasma membrane receptors for induction of intracellular signaling pathways, but also bypass intracellular oncogenic impasses to induce cell death by regulated mechanisms. Based on work reviewed here, a likely scenario for cell and tumor demise includes nsPEF-induced permeabilization of the plasma membrane, $\mathrm{Ca}^{2+}$ influx, dissipation of the mitochondrial membrane potential, which is likely due to events beyond permeabilization of the inner mitochondrial membrane, cytochrome $\mathrm{c}$ release and activation of caspase-dependent and -independent cell death mechanisms. in vivo, nsPEF-treated orthotopic rat N1-S1 hepatocellular carcinoma tumors exhibit caspase- 9 and caspase-3 positive and -negative tumor cells, indicating intrinsic apoptotic and non-apoptotic cell death. Interestingly, after N1-S1 tumor ablation and clearance, rats are resistant to challenge injections of the same N1-S1 tumor cells, indicating a protective, vaccine-like effect that appears to be due to innate and/or adaptive immune responses that are under further investigation. This provides additional impetus to further develop nsPEF ablation as a cancer therapy
\end{abstract}

Keywords: Electric fields, Calcium, Mitochondria membrane potential, Mitochondria permeability transition pore, Cytochrome c, Caspases, Apoptosis, Non-apoptotic cell death, Hepatocellular carcinoma, Vaccine-like effect
Volume 2 Issue I - 2015

\author{
Stephen Beebe J \\ Frank Ready Research Center for Bioelectrics, USA
}

Correspondence: Stephen Beebe J, Frank Ready Research Center for Bioelectrics, 42II Monarch Way, IRP, II Room 34I, 235I0,Virginia, USA, Tel (757) 683-2405, Fax (757) 45I-1010 Email sbeebe@odu.edu

Received: October 12,2014 | Published: January 02, 2015
Abbreviations:NsPEFs, Nanosecond Pulsed Electric Fields; mPTP, Mitochondria Permeability Transition Pore; VDAC, Voltage Dependent Anion Channel; ANT, Adenine Nucleotide Transporter; DISC, Death-Induced Signaling Complex; IRE, Irreversible Electroporation; HCC, Hepatocellular Carcinoma

\section{Introduction}

A major problem that cancer therapeutics faces today is coping with a diversity of cancer diseases. Hanahan and Weinberg. ${ }^{1,2}$ reasoned that since all mammalian cells express the same molecular mechanisms for proliferation, differentiation and death, cancers should share a limited number of systems that govern their behavior. This is insightful because cancers represent an array of diseases. To develop cancer research into a more logical science and to provide a focused characterization of cancer, Hanahan and Weinberg. ${ }^{1}$ defined six major hallmarks of cancer that control cell homeostasis and proliferation. These include self-sufficiency in growth signals, insensitivity to growth-inhibitory (antigrowth) signals, evasion of apoptosis, limitless replicative potential, sustained angiogenesis, and tissue invasion and metastasis. Progress made in the last decade has added two emerging hallmarks including reprogramming of energy metabolism and evading immune destruction. ${ }^{2}$ More recently Vogelstein et al. ${ }^{3}$ defined a cancer genome landscape, finding that cancers are "pathway diseases" with two to eight "driver gene" mutations from a class of 12 signaling pathways that regulate three core cellular processes: cell fate, cell survival and genome maintenance. With an evolving understanding of molecular mechanisms of cancer, treatment strategies using mutationspecific small molecule drugs or monoclonal antibodies have been developed to specifically target some cancers in the perspective of defined cancer hallmarks. One example is the treatment of B-RAF oncoprotein in melanoma, which provides sufficiency of growth signals. ${ }^{4}$ However, tumors treated with B-RAF inhibitors nearly always develop resistance after about six to nine months of treatment, and the tumors return or begin growing again. Thus, new treatment modalities to treat cancer are needed. When these new modalities are introduced it should be of interest to determine which of the hallmarks of cancer are targeted. Furthermore, increasing evidence indicates that it is important to know mechanisms of cell death.

During developmental and homeostatic cell death, apoptosis is anti-inflammatory and immunologically silent. However, a number of recent studies indicate that caspase-dependent processes are important for immunogenicity. ${ }^{5}$ In chemotherapy-induced cell death, some (anthracyclins), but not all (mitomycin C) caspase-inducing stimuli initiates' immunogenic cell death. ${ }^{6}$ thus immunogenicity depends on factors other than caspase activation. Apoptosis has been shown to induce maturation of dendritic cells leading to T-cell activation and immunity. ${ }^{7}$ and apoptotic cells not only undergo degradation, but also deliver processed antigen to dendritic cells for cross-presentation. ${ }^{8}$ Immunogenic cell death has obvious advantages for cancer treatment.

There are advantages and disadvantages to investigating cell death mechanisms both in vitro and in vivo and in a word they are reciprocal. in vitro experiments with cells allow more flexibility in experimental designs, but they exclude systems of the entire living organism. in vivo studies include systems of the entire living organism, but they allow much less flexibility in experimental designs. Thus, approaches should include both experimental strategies and determine whether or not in vitro results translate into in vivo realities.

Over the last decade, a novel cancer treatment strategy makes use of pulse power technology by applying nanosecond pulsed electric fields (nsPEFs) to cancer cells and tumors. Pulse power compresses electric energy and releases it in sub micro second bursts that achieve high power and low energy. When a stored joule of energy is released all at once in one second, the peak power delivered is only 1 watt $\left(10^{1}\right)$. When it is released in a microsecond, the power is one megawatt $\left(10^{6}\right)$; when it is release in one nanosecond the peak power is one 
giga watt $\left(10^{9}\right)$. NsPEFs cause cell membrane supra-electroporation resulting in high density "nanopores" (nm diameter) in all cellular membranes. ${ }^{9,10}$ Vernier et al. ${ }^{11}$ demonstrated that nanopores facilitated voltage-driven phosphatidyl serine translocation in lipid bilayers and Pakhomov et al. ${ }^{12}$ identified lipid nanopores in cell membranes that formed stable, ion channel-like conduction pathways. This is a unique transition of a technology used in high power physics to applications in biology and medicine. In this brief discussion, an overview of in vitro and in vivo results using nsPEFs is presented as an alternative therapy for cancer treatment.

\section{Some lessons learned from in vitro experiments}

A series of studies highlighted here provided insight into effects of nsPEFs on plasma membranes, mitochondria and cell death mechanisms. In one series of studies we looked at effects of nsPEFs on plasma membranes as $\mathrm{Ca}^{2+}$ influx using Fluo-4 and on intracellular organelles as changes in the mitochondria membrane potential $(\Delta \Psi \mathrm{m})$ with TMRE. We used 4 different nsPEF waveforms that differed in their rise-fall times and bipolar components at the end of the pulse (bipolar tails). ${ }^{13}$ These studies are based on the hypothesis that high frequency components are important for intracellular effects of nsPEFs. ${ }^{14}$ In another series, we looked at activation of caspase-3, -9 and -8 and cell death in Jurkat cell mutants that were deficient in key protein in extrinsic (FADD, caspase-8) and intrinsic (APAF1) apoptosis pathways. ${ }^{15}$ Thus, the following results are a review of mostly recent work from the Beebe laboratory. ${ }^{13-22}$

NSPEF induce plasma membrane poration at lower electric fields than that required for loss of the mitochondria membrane potential $(\Delta \Psi \mathrm{m})$, indicating that nsPEFs affect plasma membranes more readily that intracellular membranes or organelles. High frequency components of nsPEFs (frequency in $\mathrm{Hz}=1 /$ time in sec) are most effective to dissipate the mitochondria membrane potential $(\Delta \Psi \mathrm{m})$, but are less important for plasma membrane permeabilization using $\mathrm{Ca}^{2+}$ influx as a marker. Thus, the pulse waveform appears to selectively affect $\Delta \Psi \mathrm{m}$ and possibly other intracellular components. Cell death is closely correlated with loss of $\Delta \Psi \mathrm{m}$; high intracellular $\mathrm{Ca}^{2+}$ is necessary, but not sufficient for cell death. Thus, nsPEFinduced cell death requires both influx of extracellular $\mathrm{Ca}^{2+}$ and dissipation of $\Delta \Psi \mathrm{m} \cdot{ }^{20,21}$ This is consistent with a "two hit" hypothesis for cell death. ${ }^{23,24}$ At electric fields sufficient to induce cell death in $40-60 \%$ of cells, dissipation of $\Delta \Psi \mathrm{m}$ is calcium-dependent. At electric fields that induce cell death in $90 \%$ of cells, dissipation of $\Delta \Psi \mathrm{m}$ is partially $\mathrm{Ca}^{2+}$-independent. This suggests two different thresholds for nsPEF effects on $\triangle \Psi \mathrm{m}$, the higher threshold, but not the lower one, is most likely permeabilization of the inner mitochondrial membrane. Plasma membrane permeabilization is not $\mathrm{Ca}^{2+}$ dependent, suggesting effects on $\Delta \Psi \mathrm{m}$ at the lower threshold are due to events other than permeabilization. Since essentially all $\mathrm{Ca}^{2+}$-mediated effects depend on a protein(s), nsPEFs-induced dissipation of $\Delta \Psi \mathrm{m}$ may include effects on a protein or protein complex [20,21]. Showing a direct electric field effect on a protein, nsPEFs inactivated the catalytic subunit of the cAMP-dependent protein kinase (PKA). ${ }^{22}$ The most likely protein complex for a nsPEF effect on $\triangle \Psi \mathrm{m}$ is the mitochondria permeability transition pore (mPTP) complex. However, inhibitors of components of the mPTP complex or associated proteins, including cyclophilin $\mathrm{D}$, voltage dependent anion channel (VDAC) and adenine nucleotide transporter (ANT), had no effect on nsPEF-induced loss of $\Delta \Psi \mathrm{m} .{ }^{22}$ Furthermore, over expression of Bcl-xl, which binds to VDAC and attenuated heat stress- and genotoxic-induced apoptosis in the same Jurkat clone. ${ }^{25,26}$ had no effect on nsPEF-induced dissipation of $\Delta \Psi \mathrm{m}$ or cell death [Beebe, unpublished].
Using Jurkat clones that were deficient in FADD and caspase-8, abolishing formation of the death-induced signaling complex (DISC) and other FADD complexes, did not prevent nsPEF-induced cell death, indicating that FADD and the DISC was not necessary for nsPEFinduced cell death. ${ }^{15}$ However, cell survival in U937 cells and Jurkat cells following $10 \mathrm{~ns}$ pulse exposures depends on extrinsic apoptotic regulators cFLIP and FAS ligand, respectively. ${ }^{27}$ Using a Jurkat clone that was APAF1-deficient demonstrated that nsPEF-induced cell death (ten $60 \mathrm{~ns}$ pulses) is caspase-dependent at intermediate electric fields $(20-40 \mathrm{kV} / \mathrm{cm})$ and caspase-independent at high electric fields $(\geq 50$ $\mathrm{kV} / \mathrm{cm}$ ), underscoring that in response to nsPEFs, apoptosis is not the only executed form of cell death. ${ }^{15}$ Cells can be driven into cell death subroutines that include necrotic features. ${ }^{28,29} \mathrm{Cal}$ pains, which are activated by nsPEFs. ${ }^{20,30}$ have been shown to be involved in calpaindependent necrotic and/or apoptosis-like programmed cell death. ${ }^{31}$ These data indicate that oncogenic mechanisms that block cell death by over expressing of Bcl-xl, blocking the DISC or FADD complexes or preventing caspase activation can be bypassed by nsPEF ablation.

\section{Cancer therapy with electric fields}

Approaches using electric fields as cancer therapy include electrochemotherapy delivering poorly permeable chemotherapeutic agents by electroporation. ${ }^{32,33}$ or electroporation to deliver DNA. ${ }^{34}$ These topics have been recently reviewed. ${ }^{35,36}$ Another therapy based on uses of electric fields is irreversible electroporation (IRE), which increases electric fields to produce cell necrosis through irreversible plasma cell membrane defects. ${ }^{37}$

\section{Treatment of Cancer using Pulsed Power with NsPEFs}

Another extension of conventional electroporation takes greater advantage of pulsed power technology with nsPEFs, which continues to be explored for tumor ablation. Within the last decade, applications of ultra-high pulse power have been extended to biology, medicine and cancer therapy. ${ }^{19,38}$ as indicated by successful treatment of mouse B16 melanoma. ${ }^{39,40}$ a human basal cell carcinoma. ${ }^{41}$ and mouse ectopic hepatocellular carcinoma (HCC). ${ }^{42}$ More recently, an orthotopic rat N1S1 HCC has been successfully treated. ${ }^{43,44}$ Efficacy of nsPEFs has been demonstrated for murine basal cell carcinoma. ${ }^{45}$ and human pancreatic carcinoma in a murine xenograft model [46 It has also shown in the first human trial that nsPEFs or nano-electro-ablation eliminated 7 of 10 basal cell carcinomas, while 2 partially regressed. After treatment two of the 7 exhibited seborrheic keratosis in the absence of basaloid cells and one treated lesion recurred by week 10 and histologically had the appearance of a squamous cell carcinoma. These treatments left no visible scars at the treatment sites [47].

\section{Treatment of Orthotopic Rat NISI HCC with nsPEFs}

N1-S1 HCC tumors were initiated and treated 7 days later when tumors were about $55 \times 55 \mathrm{~mm}$. NsPEF treatments were delivered to tumor tissue using a 5 needle electrode array [44]. Treatments included pulses with $100 \mathrm{~ns}$ durations, electric field strengths of $50 \mathrm{kV} / \mathrm{cm}$ and various pulse numbers from 100 to 1000 with repetition rates of 1 Hz. Tumor growth was followed by ultrasound. Rats were euthanized when heavy tumor burden was detected or at study end points. Tumors were weighed and volumes were determined or classified as complete response if no significant masses were observed; as a partial response if tumors were small, but still growing slowly; as stable, if tumors were present but not growing; and no response if tumors grew so large that euthanasia was required due to tumor burden.

Tumors that were treated with 100,300 or 500 pulses were not visible at 2 weeks, but by 6 weeks had begun to grow again. In a large 
study, tumors received either sham treatment or 1000 pulses, 100 $\mathrm{ns}, 50 \mathrm{kV} / \mathrm{cm}$ at $1 \mathrm{~Hz}$. Greater than $80 \%$ of tumors were completely eliminated. Two tumors showed partial responses, one tumor was stable and three others continued to grow albeit slowly without a response. Sham-treated tumors readily grew, eventually requiring euthanasia due to tumor burden. Active caspases-3 and -9 were present in tumors 1, 2, 4 and $6 \mathrm{~h}$ after treatment, but not in shamtreated tumors. Active caspase- 8 was not readily identified at any time points. By $6 \mathrm{~h}$ after treatment, about $50 \%$ and $40 \%$ of cells showed active caspase- 3 and -9 , respectively. Not all cells expressed active caspases indicating that not all cells die by apoptosis.

In another large study, tumors ablated with 1000 pulses with 100 $\mathrm{ns}, 50 \mathrm{kV} / \mathrm{cm}$ and at $1 \mathrm{~Hz}$ were followed by challenge injections of the same cells 7 weeks after treatment. Twenty animals were sham-treated and 23 were treated with nsPEFs. Within 40 days 21 or $91.3 \%$ of rats were tumor free while all sham-treated rats required euthanasia due to tumor burden. The 21 surviving rats were then challenged with a second injection identical to the initial one in either the same lobe that carried the tumor or an adjacent right lobe. All 21 rats with nsPEFablated tumors remained tumor free for as long as 20 weeks, when the study was terminated. In contrast, 24 age-matched, naïve control rats (shipping mates of those initially treated) required euthanasia between 4-5 weeks after injection due to tumor burden. Thus a vaccine-like, protective affect is evident which prevents recurrence after complete nsPEF ablation.

\section{Possible advantages for nspef ablation as a cancer therapy}

There may be several advantages for using nsPEF ablation as a means for cancer therapy as opposed to other physical methods that rely on overt necrosis for tumor cell death. One advantage is that nsPEFs target multiple programmed cell death mechanisms for apoptosis induction and anti-angiogenesis, two well-known cancer hallmarks, the latter necessary for a third cancer hallmark, invasion and metastasis. ${ }^{40}$ In addition, the treatment is rapid, targeting multiple death mechanisms with minimal treatment exposures, which reduces chances for resistances and recurrences. The electric fields also target mitochondria-mediated programmed cell death, which can bypass many cancer-causing mutations. While nsPEFs exhibit some cell specificity, with high electric fields, a broad specificity for cell death induction occurs in the tumor mass and the microenvironment, including rapidly growing tumor cells, slower growing host cells that collaborate with tumor cells and cancer stem cells (the latter has not been shown directly). The treatment causes local infarction of small vessels, depriving tumors of feeder vessels, which disrupts nutrient and oxygen supplies inducing local stresses with minimal local and systemic side effects. Finally there is a possibility that nsPEFs enhance immune surveillance from cells undergoing apoptosis. There is accumulating evidence that the presence of apoptotic cells is advantageous for antigen presentation. ${ }^{6,7,47-50}$ Surface exposure of immunogenic effectors is facilitated during early apoptosis, caspase activation and endoplasmic reticulum stress. This is followed by the release of soluble factors that are indispensable for effective immune response. ${ }^{51}$

\section{Conclusion}

NsPEFs induce both caspase-dependent (apoptosis) and caspaseindependent cell death in tumor cells and tissues through pathways that appear to be mediated, at least in part, by intrinsic mechanisms. It is clear that apoptosis is not the sole form of cell death executed in response to nsPEFs and that different nsPEF conditions may induce different mechanisms for cell demise. Two prominent events that lead to cell death are influx of $\mathrm{Ca}^{2+}$ through permeabilized plasma membranes and dissipation of the $\Delta \Psi \mathrm{m}$ by a mechanism(s) that may include nsPEF-induced effects on a protein(s). Caspasedependent (apoptotic) and caspase-independent cell death may enhance immunogenic cell death that can possibly generate inherent resistances to further development of the treated type of cancer, mimicking a vaccine effect.

\section{Acknowledgments}

None.

\section{Conflicts of interest}

None.

\section{References}

1. Hanahan D, Weinberg RA The hallmarks of cancer. Cell. 2000;100(1):57-70.

2. Hanahan D, Weinberg RA Hallmarks of cancer: the next generation. Cell. 2011;144(5):646-674.

3. Vogelstein B, Papadopoulos N, Velculescu VE et al. Cancer genome landscapes. Science. 2013;339(6127):1546-1558.

4. Mercer KE, Pritchard CA RAF proteins and cancer: B-Raf is identified as a mutational target. Biochim Biophys Acta. 2003:1653(10):25-40.

5. Kroemer G, Galluzzi L, Kepp O et al. Immunogenic cell death in cancer therapy. Annu Rev Immunol. 2013;31:51-72.

6. Casares N, Pequignot MO, Tesniere A et al. Caspase-dependent immunogenicity of doxorubicin-induced tumor cell death. J Exp Med. 2005;202(12):1691-1701

7. Albert ML, Pearce SF, Francisco LM et al. Immature dendritic cells phagocytose apoptotic cells via alphavbeta5 and CD36, and cross-present antigens to cytotoxic T lymphocytes. $J$ Exp Med. 1998;188(7):1359-1368.

8. Blachere NE, Darnell RB, Albert ML Apoptotic cells deliver processed antigen to dendritic cells for cross-presentation. PLoS Biol. 2005;3(6):185.

9. Stewart DA, Gowrishankar TR, Weaver JC Transport lattice approach to describing electroporation: use of local asymptotic model. IEEE Trans Plasma Sci. 2004;32:1696-1708.

10. Gowrishankar TR, Esser AT, Vasilkoski Z et al. Microdosimetry for conventional and supra-electroporation in cells with organelles. Biochem Biophys Res Commun. 2006;341(4):1266-1276.

11. Vernier PT, Ziegler MJ, Sun Y et al. Nanopore-facilitated, voltagedriven phosphatidyl serine translocation in lipid bilayers - in cells and in silico. Phys Biol. 2006;3(4):233-247.

12. Pakhomov AG, Bowman AM, Ibey BL et al. Lipid nanopores can form a stable, ion channel-like conduction pathway in cell membrane. Biochem Biophys Res Commun. 2009;385(2):181-186.

13. Beebe SJ, Chen YJ, Sain NM et al. Transient features in nanosecond pulsed electric fields differentially modulate mitochondria and viability. PLoS One. 2012;7(12):51349.

14. Schoenbach KH, Beebe SJ, Buescher ES Intracellular effect of ultra short electrical pulses. Bioelectromagnetics. 2001;22(6):440-448.

15. Ren W, Sain NM, Beebe SJ Nanosecond pulsed electric fields (nsPEFs) activate intrinsic caspase-dependent and caspase-independent cell death in Jurkat cells. Biochem Biophys Res Commun. 2012;421(4):808-812.

16. Beebe SJ, Fox PM, Rec LH et al. Nanosecond Pulsed Electric Field (nsPEF) Effects on Cells and Tissues: Apoptosis Induction and Tumor Growth Inhibition. Plasma Science, IEEE Transactions. 2002;30(1):286-292. 
17. Beebe SJ, Fox PM, Rec LJ et al. Nanosecond, high-intensity pulsed electric fields induce apoptosis in human cells. FASEB J. 2003;17(11):1493-1495.

18. Beebe SJ, Schoenbach KH Nanosecond pulsed electric fields: a new stimulus to activate intracellular signaling. J Biomed Biotechnol. 2005;2005(4):297-300.

19. Beebe SJ Bioelectrics in Basic Science and Medicine: Impact of Electric Fields on Cellular Structures and Functions. J Nanomedic Nanotechnol. 2012;4:163.

20. Beebe SJ, Sain NM, Ren W Induction of Cell Death Mechanisms and Apoptosis by Nanosecond Pulsed Electric Fields (nsPEFs). Cells. 2013;2(1):136-162.

21. Beebe SJ Cell respo nses without receptors and ligands using nanosecond pulsed electric fields (nsPEFs). Int J Nanomedicine. 2013;8:3401-3404.

22. Beebe SJ Considering effects of nanosecond pulsed electric fields on proteins. Bioelectrochemistry. 2014;S1567-S5394(14)00130-3.

23. Jacobson J, Duchen MR Mitochondrial oxidative stress and cell death in astrocytes-requirement for stored $\mathrm{Ca} 2+$ and sustained opening of the permeability transition pore. J Cell Sci. 2002;115(6):1175-1188.

24. Brookes PS, Yoon Y, Robotham JL et al. Calcium, ATP, and ROS a mitochondrial love-hate triangle. Am J Physiol Cell Physiol. 2004;287(4):817-833.

25. Shawgo ME, Shelton SN, Robertson JD Caspase-mediated Bak activation and cytochrome c release during intrinsic apoptotic cell death in Jurkat cells. J Biol Chem. 2008;283(51):35532-35538.

26. Shelton SN, Dillard CD, Robertson JD Activation of caspase-9, but not caspase-2 or caspase- 8 , is essential for heat-induced apoptosis in Jurkat cells. J Biol Chem. 2010;285(52):40525-40533.

27. Estlack LE, Roth CC, Thompson GL $3^{\text {rd }}$ et al. Nanosecond pulsed electric fields modulate the expression of Fas/CD95 death receptor pathway regulators in U937 and Jurkat Cells. Apoptosis. 2014;19(2):1755-1768.

28. Pakhomova ON, Gregory BW, Semenov I et al. Two modes of cell death caused by exposure to nanosecond pulsed electric field. PLoS One. 2013;8(7):70278.

29. Morotomi-Yano K, Akiyama H, Yano K Different involvement of extracellular calcium in two modes of cell death induced by nanosecond pulsed electric fields. Arch Biochem Biophys. 2014;555-556:47-54.

30. Ren W, Beebe SJ An apoptosis targeted stimulus with nanosecond pulsed electric fields (nsPEFs) in E4 squamous cell carcinoma. Apoptosis. 2011;16(4):382-393.

31. Nikoletopoulou V, Markaki M, Palikaras K et al. Crosstalk between apoptosis, necrosis and autophagy. Biochim Biophys Acta. 2013;1833(12):3448-3459.

32. Mir LM, Orlowski S, Belehradek J, Paolett C Electro chemotherapy potentiation of antitumor effect of bleomycin by local electric pulses. Eur J Cancer. 1991;27(1):68-72.

33. Heller R, Jaroszeski MJ, Glass LF et al. Phase I/II trial for the treatment of cutaneous and subcutaneous tumors using electro chemotherapy. Cancer. 1996;77(5):964-971.

34. Neumann E, Schaefer-Ridder M, Wang Y et al. Gene transfer into mouse lyoma cells by electroporation in high electric fields. EMBO J
1982;1(7):841-845.

35. Heller LC Heller R in vivo electroporation for gene therapy. Hum Gene Ther. 2006;17(9):890-897.

36. Beebe SJ, Schoenbach KH, Heller R Bioelectric Applications for Analysis and Treatment of Melanoma. Cancers (Basel). 2010;2(3):1731-1770.

37. Davalos RV, Mir IL, Rubinsky B Tissue ablation with irreversible electroporation. Ann Biomed Eng. 2005;33(2):223-231.

38. Beebe SJ, Ford WE, Ren W, Chen X Pulse power ablation of melanoma with nanosecond pulsed electric fields, In: R Morton (Ed.), Treatment of Metastatic Melanoma, In Tech Croatia, Croatia, pp. 2011;231-268.

39. Nuccitelli R, Chen X, Ford W et al. Nanosecond pulsed electric fields cause melanomas to self-destruct. Biochem Biophys Res Commun. 2006;343(2):351-360.

40. Chen X, Kolb JF, Swanson RJ et al. Apoptosis initiation and angiogenesis inhibition: melanoma targets for nanosecond pulsed electric fields. Pig Cell \& Melanoma Res. 2010;23(4):554-563.

41. Garon EB, Sawcer D, Vernier PT et al. in vitro and in vivo evaluation and a case report of intense nanosecond pulsed electric field as a local therapy for human malignancies. Int J Cancer. 2007;121:675-682.

42. Chen X, Zhuang J, Kolb JF et al. Long term survival of mice with hepatocellular carcinoma after pulse power ablation with nanosecond pulsed electric fields. Technol Cancer Res Treat. 2012;11(1):83-93.

43. Chen R, Chen X, Beebe SJ Nanosecond Pulsed Electric Field (nsPEF) Ablation as an Alternative or Adjunct to Surgery for Treatment of Cancer. Surgery: Current Research. 2013;S12:005.

44. Chen R, Sain NM, Harlow KT et al. A protective effect after clearance of orthotopic rat hepatocellular carcinoma by nanosecond pulsed electric fields. Eur J Cancer. 2014;50(15):2705-2713.

45. Nuccitelli R, Tran K, Athos B et al. Nanoelectroablation therapy for murine basal cell carcinoma. Biochem BiophysRes Commun. 2012;424(3):446-450.

46. Nuccitelli R, Huynh J, Lui K et al. Nanoelectroablation of human pancreatic carcinoma in a murine xenograft model without recurrence. Int J Cancer. 2013;132(8):1933-1939.

47. Nuccitelli R, Wood R, Kreis $M$ et al. First-in-human trial of nanoelectroablation therapy for basal cell carcinoma: proof of method. Exp Dermatol. 2014;23(2):135-137.

48. Russo V, Tanzarella S, Dalerba $\mathrm{P}$ et al. Dendritic cells acquire the MAGE-3 human tumor antigen from apoptotic cells and induce a class I-restricted $\mathrm{T}$ cell response. Proc Natl Acad Sci USA. 2000;97(5):2185-2190.

49. Chattergoon MA, Kim JJ, Yang JS et al. Targeted antigen delivery to antigen-presenting cells including dendritic cells by engineered Fasmediated apoptosis. Nat Biotechnol. 2000;18(9):974-979.

50. Savill J, Dransfield I, Gregory C, Haslett C A blast from the past: clearance of apoptotic cells regulates immune responses. Nat Rev Immunol. 2002;2(12):965-975.

51. Kepp O, Tesniere A, Zitvogel L, Kroemer G The immunogenicity of tumor cell death. Curr Opin Oncol. 2009;21(1):71-76. 